\title{
Studies on Heterosis for Yield and Yield Contributing Characters in Ridge Gourd (Luffa acutangula L.)
}

\author{
D. G. Wakale, V. S. Jagtap and O. A. Sogam*
}

Dept. of Horticulture, College of Agriculture, Latur, Vasantrao Naik Marathwada Krishi Vidyapeeth Parbhani, M.S. (431 402), India

\section{Corresponding Author}

O. A. Sogam

e-mail: onkarsogam@gmail.com

\author{
Article History \\ Article ID: 3C0585b \\ Received in $26^{\text {th }}$ October, 2017 \\ Received in revised form $20^{\text {th }}$ August, 2018 \\ Accepted in final form $31^{\text {st }}$ August, 2018
}

\begin{abstract}
Half diallel analysis was carried out during kharif- 2016 at Instructional-Cum-Research Farm, Department of Horticulture, College of Agriculture, Latur, Vasantrao Naik Marathwada Krishi Vidyapeeth, Parbhani to study the heterosis per cent for yield and yield contributed characters. The $21 \mathrm{~F}_{1}$ hybrids along with their seven parent ware evaluated. Observation were recorded on five randomly selected tagged plant from each treatment for yield and yield attributing character viz., length of vine, no. of nodes plant ${ }^{-1}$, length of fruit, diameter of fruit, no. of fruit vine ${ }^{-1}$, wt. of fruit, no. of fruit vine ${ }^{-1}$, wt. of fruit, wt. of fruit vine ${ }^{-1}$, fruit yield ha ${ }^{-1}$. The values of $F_{1}$ averaged over replications were used for estimating heterosis. Manifestation of heterosis of higher order for number of fruit vine ${ }^{-1}$, length of fruit, weight of fruit, weight of fruit vine ${ }^{-1}$ and fruit yield ha-1. The maximum heterosis over better parent for fruit yield ha-1 was observed to be $38.58 \%$ and that over useful heterosis was found $84.42 \%$. Three potential heterosis cross combinations viz. Arka Sujat $\times$ Krishna- 51 , Krishna-51×Kranti-30 and Krishna-51×Swati Green were identified as for promising for fruit yield per ha ${ }^{-1}$ The significant heterosis crosses involved high $\times$ high, low $\times$ high, low $\times$ low and high $\times$ low performance of parents. They have also exhibited higher additive variance. Hence, they may be exploited for development of hybrid in ridge gourd.
\end{abstract}

Keywords: Ridge gourd (Luffa acutangula L.), genotype, heterosis

\section{Introduction}

Ridge gourd (Luffa acutangula L.) is one of the popular vegetable crops belonging to the family cucurbitaceae. It is extensively cultivated throughout India; but it is more popular in south and east part of India. Ridge gourd has good nutritive value. Heterosis breeding has been a recognized practical tool in providing the breeder a means for increasing the yield and other economic traits.

The hybrid vigour or the superiority of the hybrids $\left(F_{1}\right)$ over parents may be manifested in terms of high productivity, uniformity, improved quality, built in resistance, environmental condition, earliness etc. However, it never happens that each hybridization is accompanied by the manifestation of hybrid vigour. Only certain pair of parents gives heterotic progeny. Therefore, for development of an effective heterosis breeding programme in ridge gourd one needs to elucidate the genetic variance nature and magnitude of quantitively inherited traits and estimate parents in hybrid combinations. The hybrid vigour is at the maximum in hybrid $\left(F_{1}\right)$. The attempts of commercial production of hybrids $\left(F_{1}\right)$ in vegetables in general and the cucurbits in particular was started as a early as 1935 in Japan and 1940 in USA ( Singh and Swarup, 1971) .

Heterosis for yield and yield contributing characters has been reported in ridge gourd by Mole et al. (2001), Hedau and Sirohi (2004), Naliyadhara et al. (2007), Karmakar (2011), Kumar et al (2011) Narasannavar et al. (2014), Radha Rani (2014) and Kannan et al. (2015).

\section{Materials and Methods}

The inbred lines of seven genotypes namely Kokan Harita $\left(P_{1}\right)$, Arka sujat $\left(P_{2}\right)$, Pusa Nasdar $\left(P_{3}\right)$, Krishna-51 $\left(P_{4}\right)$, Jaipuri $\left(P_{5}\right)$, Krishna-30 $\left(P_{6}\right)$, Swati Green $\left(P_{7}\right)$ were selected for the purpose of crossing programme and sown in crossing block at Instructional-Cum Research Farm, Department of Horticulture, College of Agriculture, Latur. The $21 \mathrm{~F}_{1}$ hybrids along with their seven parent ware evaluated. Observation were recorded on five randomly selected tagged plant from each treatment for yield and yield attributing character viz. ,length of vine, no. of nodes plant ${ }^{-1}$, length of fruit, diameter of fruit, no. of fruit vine ${ }^{-1}$, wt. of fruit, no. of fruit vine ${ }^{-1}$, wt. of fruit, wt. of fruit vine ${ }^{-1}$, fruit yield ha-1. The values of $F_{1}$ averaged over replications were used for estimating heterosis. The magnitude of heterosis was calculated as percentage increase or decrease of $F_{1}$ mean over the mean of better parent (BP) (Turner, 1953 and Hays et al. (1955) and per cent superiority over standard hybrid check were calculated. The analysis of variance, for all traits under study, was carried out 
the method suggested by Panse and Sukhatme (1985).

\section{Result and Discussion}

Analysis of variance for yield and yield component in Table 1.
The treatment means were further sub divided in to parents, crosses and parent versus crosses. The parents showed significant differences for the characters like length of vine, days required to first harvest, length of fruit, diameter of fruit,

\begin{tabular}{|c|c|c|c|c|c|c|c|c|c|c|c|}
\hline Source & d.f. & $\begin{array}{l}\text { Length of } \\
\text { vine }(\mathrm{cm})\end{array}$ & $\begin{array}{l}\text { No. of } \\
\text { nodes } \\
\text { plant }^{-1}\end{array}$ & $\begin{array}{l}\text { No. of } \\
\text { female } \\
\text { flower } \\
\text { vine }^{-1}\end{array}$ & $\begin{array}{c}\text { Days re- } \\
\text { quired } \\
\text { to first } \\
\text { harvest }\end{array}$ & $\begin{array}{l}\text { Length } \\
\text { of fruit } \\
(\mathrm{cm})\end{array}$ & $\begin{array}{l}\text { Diameter } \\
\text { of fruit } \\
(\mathrm{cm})\end{array}$ & $\begin{array}{l}\text { No. of } \\
\text { fruits } \\
\text { vine }^{-1}\end{array}$ & $\begin{array}{l}\text { Wt.of } \\
\text { fruit } \\
\text { (g) }\end{array}$ & $\begin{array}{l}\text { Wt. of } \\
\text { fruit } \\
\text { vine }^{-1} \\
\text { (kg) }\end{array}$ & $\begin{array}{l}\text { Fruit } \\
\text { yield ha-1 } \\
\text { (q) }\end{array}$ \\
\hline Treatment & 27 & $4687.57^{* *}$ & $6.456^{* *}$ & $5.44^{* *}$ & $4.85^{* *}$ & $20.95^{* *}$ & 0.087 & $3.89^{* *}$ & $282.64^{* *}$ & $0.17^{* *}$ & $3285.31^{* *}$ \\
\hline Parent & 6 & $2930.47^{* *}$ & 0.472 & 3.60 & $5.11^{* *}$ & $11.22^{*}$ & $0.096^{* *}$ & 1.13 & $266.42^{* *}$ & $0.27^{* *}$ & $3346.03^{* *}$ \\
\hline Crosses & 20 & $5449.00^{* *}$ & $8.436^{* *}$ & $4.28^{* *}$ & $4.98^{* *}$ & $23.91^{* *}$ & $0.088^{*}$ & $3.25^{* *}$ & $295.45^{* *}$ & $0.14^{* *}$ & $3276.40^{* *}$ \\
\hline $\mathrm{P} \times \mathrm{C}$ & 1 & $22562.71^{* *}$ & 2.751 & $39.72^{* *}$ & 0.72 & $20.06^{* *}$ & $1.94^{* *}$ & $33.17^{* *}$ & $123.58^{* *}$ & 0.10 & $3099.18^{* *}$ \\
\hline Error & 27 & 174.10 & 2.269 & 2.08 & 1.26 & 3.19 & 0.047 & 0.86 & 1.23 & 0.06 & 276.38 \\
\hline
\end{tabular}

weight of fruit, wt. of fruit vine ${ }^{-1}$ and wt. of fruit ha ${ }^{-1}$ and other characters are non-significant. The crosses showed significant for all characters.

Per cent heterosis over better parent and standard hybrid in Table 2. The cross combination Kokan Harita×Kranti-30 (30.70\%) exhibited highest positive significant heterosis over better parents and the cross combination Kokan HaritaxJaipuri $(39.86 \%)$ recorded significantly highest positive heterosis over

\begin{tabular}{|c|c|c|c|c|c|c|c|c|c|c|}
\hline \multirow[t]{2}{*}{ Parent/ Crosses } & \multicolumn{2}{|c|}{ Length of vine $(\mathrm{cm})$} & \multicolumn{2}{|c|}{$\begin{array}{l}\text { No. of nodes } \\
\text { plant }^{-1}\end{array}$} & \multicolumn{2}{|c|}{$\begin{array}{l}\text { No. of female } \\
\text { flower vine }{ }^{-1}\end{array}$} & \multicolumn{2}{|c|}{$\begin{array}{l}\text { Days required } \\
\text { to first harvest }\end{array}$} & \multicolumn{2}{|c|}{$\begin{array}{l}\text { Length of fruit } \\
(\mathrm{cm})\end{array}$} \\
\hline & BP & $\mathrm{SH}$ & BP & $\mathrm{SH}$ & BP & $\mathrm{SH}$ & BP & $\mathrm{SH}$ & BP & $\mathrm{SH}$ \\
\hline Kokan Harita×Arka Sujat & $-8.39^{*}$ & 5.49 & -5.71 & -6.13 & 16.15 & $20.28^{*}$ & -2.13 & 2.99 & $45.88^{* *}$ & $52.36^{* *}$ \\
\hline Kokan Harita×Pusa Nasdar & $29.60^{* *}$ & $37.00^{* *}$ & $10.99^{* *}$ & $10.50^{* *}$ & -0.03 & 14.12 & 0.17 & $5.97^{* *}$ & 4.80 & 11.75 \\
\hline Kokan Harita×Krishna-51 & $-20.87^{* *}$ & 1.11 & -6.15 & -6.56 & -2.92 & 13.97 & $-5.67^{* *}$ & -0.75 & 6.50 & 12.85 \\
\hline Kokan Harita×Jaipuri & $15.75^{* *}$ & $39.86^{* *}$ & $30.11^{* *}$ & $29.54^{* *}$ & $19.23^{*}$ & $22.73^{*}$ & -0.71 & $-4.48^{*}$ & $-33.68^{* *}$ & -11.42 \\
\hline Kokan Harita×Kranti-30 & $30.70^{* *}$ & $32.90^{* *}$ & $6.37^{*}$ & $5.91^{*}$ & -4.02 & -1.19 & $-4.96^{* *}$ & 3.12 & $29.83^{* *}$ & $35.60^{* *}$ \\
\hline Kokan Harita×Swati Green & $26.15^{* *}$ & $25.39^{* *}$ & -6.81 & -7.22 & 8.69 & 11.89 & $-4.96^{* *}$ & -2.12 & -14.92 & -11.14 \\
\hline Arka Sujat×Pusa Nasdar & $11.11^{* *}$ & $27.94^{* *}$ & 3.30 & 2.84 & 4.27 & $19.03^{*}$ & $-4.38^{*}$ & -2.24 & -3.43 & 2.98 \\
\hline Arka Sujat×Krishna-51 & $-25.88^{* *}$ & -5.30 & -2.86 & -3.28 & 9.86 & $28.98^{* *}$ & -2.92 & -0.75 & 15.31 & $22.19^{*}$ \\
\hline Arka Sujat×Jaipuri & $-11.63^{* *}$ & 6.78 & 3.30 & 2.84 & 11.83 & 15.81 & $-3.65^{*}$ & -1.49 & $-29.96^{* *}$ & -6.44 \\
\hline Arka Sujat×Kranti-30 & $-27.46^{* *}$ & $-16.47^{* *}$ & -2.86 & -3.28 & $17.60^{*}$ & $21.78^{*}$ & -2.19 & 1.12 & 9.77 & 7.79 \\
\hline Arka Sujat×Swati Green & $-11.15^{* *}$ & 2.31 & -4.84 & -5.25 & $25.89^{* *}$ & $30.36^{* *}$ & $-5.84^{* *}$ & $-3.73^{*}$ & $43.61^{* *}$ & $41.03^{* *}$ \\
\hline Pusa Nasdar×Krishna-51 & $-18.04^{* *}$ & 4.71 & 2.20 & 1.75 & -0.31 & 17.03 & 1.49 & 1.49 & -2.14 & 4.36 \\
\hline Pusa Nasdar×Jaipuri & $-15.40^{* *}$ & 2.23 & 0.22 & -0.22 & 1.07 & 15.38 & -1.49 & -1.49 & 12.53 & $50.31^{* *}$ \\
\hline Pusa Nasdar×Kranti-30 & $-9.75^{*}$ & -4.60 & -0.22 & -0.66 & 6.49 & $21.57^{*}$ & -0.75 & -0.75 & 7.86 & 15.02 \\
\hline Pusa Nasdar×Swati Nreen & $12.00^{* *}$ & $18.39^{* *}$ & $4.62^{* *}$ & 4.16 & -11.22 & 1.35 & -0.75 & 0.75 & 1.40 & 8.13 \\
\hline Krishna-51×Jaipuri & -5.88 & $20.26^{* *}$ & 1.76 & 1.31 & -0.94 & 16.30 & 1.50 & 1.48 & -8.53 & $22.19^{*}$ \\
\hline Krishna-51×Kranti-30 & $-23.13^{* *}$ & -1.78 & $-16.48^{*}$ & $-16.85^{*}$ & -3.03 & 13.85 & 0.75 & -2.99 & -11.68 & -6.41 \\
\hline Krishni-51×Swati Green & -6.59 & $19.35^{* *}$ & -7.47 & -7.88 & 0.21 & 17.65 & -2.26 & -0.75 & -4.03 & 1.69 \\
\hline Jaipuri×Kranti-30 & $-36.18^{* *}$ & $-22.88^{* *}$ & 2.86 & 2.41 & $32.50^{* *}$ & $32.66^{* *}$ & -2.20 & 1.20 & $-24.93^{* *}$ & 0.28 \\
\hline Jaipuri×Swati Green & $-29.56^{* *}$ & $-14.88^{* *}$ & -4.18 & -4.60 & 10.77 & 10.91 & 0.75 & 2.99 & $-28.97^{* *}$ & -5.12 \\
\hline Kranti-30 x Swati Green & 8.66 & $10.48^{*}$ & -1.76 & -2.19 & 3.35 & 0.12 & 3.76 & 2.90 & $36.80^{* *}$ & -5.12 \\
\hline
\end{tabular}




\begin{tabular}{|c|c|c|c|c|c|c|c|c|c|c|}
\hline \multirow[t]{2}{*}{ Parent/ Crosses } & \multicolumn{2}{|c|}{$\begin{array}{l}\text { Length of vine } \\
(\mathrm{cm})\end{array}$} & \multicolumn{2}{|c|}{$\begin{array}{c}\text { No. of nodes } \\
\text { plant }^{-1}\end{array}$} & \multicolumn{2}{|c|}{$\begin{array}{l}\text { No. of female } \\
\text { flower vine }{ }^{-1}\end{array}$} & \multicolumn{2}{|c|}{$\begin{array}{l}\text { Days required } \\
\text { to first harvest }\end{array}$} & \multicolumn{2}{|c|}{$\begin{array}{l}\text { Length of fruit } \\
(\mathrm{cm})\end{array}$} \\
\hline & $\mathrm{BP}$ & $\mathrm{SH}$ & B & $\mathrm{SH}$ & BP & SH & BP & SH & BP & SH \\
\hline SEd \pm & 13.19 & 13.19 & 1.5 & 1.50 & 1.44 & 1.44 & 1.12 & 1.12 & 1.78 & 1.78 \\
\hline $\mathrm{CD}(p=0.05)$ & 27.07 & 27.07 & $3 . c$ & 3.09 & 2.96 & 2.96 & 2.30 & 2.30 & 3.67 & 3.67 \\
\hline $\operatorname{CD}(p=0.01)$ & 36.55 & 36.55 & 4.1 & 4.17 & 4.00 & 4.00 & 3.11 & 3.11 & 4.95 & 4.95 \\
\hline \multicolumn{11}{|l|}{ Table 2: Continue... } \\
\hline \multirow[t]{2}{*}{ Parent/ Crosses } & \multicolumn{2}{|c|}{$\begin{array}{l}\text { Diameter of } \\
\text { fruit }(\mathrm{cm})\end{array}$} & \multicolumn{2}{|c|}{$\begin{array}{l}\text { No. of fruits } \\
\text { vine }^{-1}\end{array}$} & \multicolumn{2}{|c|}{ Wt. of fruit (g) } & \multicolumn{2}{|c|}{$\begin{array}{l}\text { Wt. of fruit } \\
\text { vine }^{-1}(\mathrm{~kg})\end{array}$} & \multicolumn{2}{|c|}{$\begin{array}{l}\text { Fruit yield ha-1 } \\
\text { (q) }\end{array}$} \\
\hline & $\mathrm{BP}$ & $\mathrm{SH}$ & $\mathrm{BP}$ & $\mathrm{SH}$ & $\mathrm{BP}$ & $\mathrm{SH}$ & $\mathrm{BP}$ & $\mathrm{SH}$ & $\mathrm{BP}$ & $\mathrm{SH}$ \\
\hline Kokan Harita×Arka Sujat & $-17.90^{*}$ & 2.11 & 2.10 & $15.05^{*}$ & $4.85^{* *}$ & $33.06^{* *}$ & $10.85^{*}$ & $64.96^{* *}$ & 4.36 & $74.71^{* *}$ \\
\hline Kokan HaritaxPusa Nasdar & -8.79 & -0.38 & 3.01 & $16.08^{*}$ & -0.51 & $26.27^{* *}$ & $-33.07^{* *}$ & -0.39 & -3.59 & $61.41^{* *}$ \\
\hline Kokan Harita×Krishna-51 & -4.57 & 4.22 & 6.99 & $20.57^{* *}$ & $2.27^{*}$ & $29.79^{* *}$ & $10.85^{*}$ & $64.96^{* *}$ & 6.33 & $78.02^{* *}$ \\
\hline Kokan Harita×Jaipuri & $8.63^{*}$ & $23.22^{* *}$ & 8.53 & $22.30^{* *}$ & $-17.56^{* *}$ & 4.63 & -21.96 & 16.14 & $-20.38^{* *}$ & $33.30^{* *}$ \\
\hline Kokan Harita×Kranti-30 & $8.44^{*}$ & $18.43^{*}$ & -3.39 & 12.21 & $-17.33^{* *}$ & 4.92 & -20.90 & 17.72 & $-23.01^{* *}$ & $28.90^{*}$ \\
\hline Kokan Harita×Swati Green & 3.69 & 13.24 & 1.47 & 14.34 & $-15.44^{* *}$ & 7.32 & -21.16 & 17.32 & $-18.62^{* *}$ & $36.24^{* *}$ \\
\hline Arka Sujat×Pusa Nasdar & 0.31 & $24.76^{* *}$ & $18.84^{*}$ & $27.74^{* *}$ & $8.93^{* *}$ & $30.26^{* *}$ & -4.93 & $36.61^{*}$ & -4.18 & $42.56^{* *}$ \\
\hline Arka Sujat×Krishna-51 & $-17.28^{*}$ & 2.88 & $47.43^{* *}$ & $53.82^{* *}$ & $10.93^{* *}$ & $32.65^{* *}$ & 5.14 & $37.01^{*}$ & $38.58^{* *}$ & $84.42^{* *}$ \\
\hline Arka Sujat×Jaipuri & -3.09 & $20.54^{*}$ & 6.87 & $17.65^{* *}$ & $7.20^{* *}$ & $28.20^{* *}$ & -4.83 & 24.02 & 14.62 & $52.53^{* *}$ \\
\hline Arka Sujat×Kranti-30 & $-22.53^{* *}$ & -3.65 & 12.21 & $30.34^{* *}$ & $-12.88^{* *}$ & 4.18 & 3.02 & 34.25 & 4.26 & $38.74^{* *}$ \\
\hline Arka Sujat×Swati Green & -10.49 & 11.32 & $18.58^{*}$ & $26.24^{* *}$ & -1.19 & $18.16^{* *}$ & -3.32 & 25.98 & 9.56 & $45.79^{* *}$ \\
\hline Pusa Nasdar×Krishna-51 & 1.49 & 4.61 & $17.45^{*}$ & $26.24^{* *}$ & $-22.17^{* *}$ & $-9.14^{* *}$ & -15.07 & 22.05 & -6.59 & $38.97^{* *}$ \\
\hline Pusa Nasdar×Jaipuri & -0.68 & 12.67 & 1.72 & 11.98 & $-9.64^{* *}$ & 6.87 & $-30.14^{*}$ & 0.39 & $-25.99^{* *}$ & 10.10 \\
\hline Pusa Nasdar×Kranti-30 & 8.01 & 11.32 & $19.00^{* *}$ & $38.22^{* *}$ & $-11.35^{* *}$ & 4.85 & -8.22 & 31.98 & -8.19 & $36.59^{* *}$ \\
\hline Pusa Nasdar×Swati Nreen & $9.17^{*}$ & 14.20 & 7.26 & $15.29^{*}$ & $-18.62^{* *}$ & $-3.74^{* *}$ & $-39.45^{* *}$ & -12.99 & $-36.34^{* *}$ & -5.30 \\
\hline Krishna-51×Jaipuri & -9.81 & 2.30 & $10.31^{*}$ & $21.43^{* *}$ & $17.87^{* *}$ & $25.93^{* *}$ & $19.41^{* *}$ & 11.42 & $-19.60^{*}$ & -5.86 \\
\hline Krishna-51×Kranti-30 & 3.17 & 6.33 & 3.12 & $19.78^{*}$ & $3.79^{* *}$ & $10.89^{* *}$ & $22.08^{* *}$ & 15.35 & $35.58^{* *}$ & $43.20^{* *}$ \\
\hline Krishni-51×Swati Green & 6.61 & 11.52 & $17.32^{*}$ & $24.90^{* *}$ & $-2.90^{* *}$ & 3.74 & 9.72 & -6.69 & $21.59^{*}$ & $37.48^{* *}$ \\
\hline Jaipuri×Kranti-30 & -13.20 & -1.54 & 3.66 & $20.41^{* *}$ & $3.74^{* *}$ & $10.40^{* *}$ & 6.25 & 0.39 & -10.61 & 4.67 \\
\hline Jaipuri×Swati Green & -4.40 & 8.45 & 1.00 & 11.19 & $11.53^{* *}$ & 8.82 & 7.12 & -6.69 & 3.26 & 20.90 \\
\hline Kranti-30 x Swati Green & 0.18 & 4.80 & -2.17 & 13.63 & 0.04 & 6.46 & 4.58 & -1.18 & -6.04 & 6.24 \\
\hline SEd \pm & 0.21 & 0.21 & 0.93 & 0.93 & 1.11 & 1.11 & 0.21 & 0.21 & 16.26 & 16.26 \\
\hline $\mathrm{CD}(p=0.05)$ & 0.44 & 0.44 & 1.90 & 1.90 & 2.27 & 2.27 & 0.44 & 0.44 & 34.11 & 34.11 \\
\hline $\mathrm{CD}(p=0.01)$ & 0.59 & 0.59 & 2.57 & 2.57 & 3.07 & 3.07 & 0.59 & 0.59 & 46.06 & 46.06 \\
\hline
\end{tabular}

standard check for length of vine Similarly result recorded by Shaha and Kale (2003), Hedau and Sirohi (2004).

The cross combinations Kokan HaritaxJaipuri (30.11\%) exhibited positively highest significant heterosis over better parents and the cross combination Kokan HaritaxJaipuri (29.54\%) recorded significantly highest positive heterosis over standard check for number of nodes vine ${ }^{-1}$. The cross combination JaipurixKranti-30 (32.50\%) exhibited highest significant heterosis over better parent and the cross combination JaipurixKranti-30 (32.66\%) recorded significantly highest positive heterosis over standard check for number of female flower. The cross combination Kokan HaritaxKrishna-51 (-5.84\%) exhibited highest significant negetive heterosis over better parents and the cross combination Kokan HaritaxJaipuri $(-4.48 \%)$ recorded significantly highest negative heterosis over standard check for days required to first harvest. Similarly result recorded by Narasannavar et al. (2014)

The cross combination Kokan HaritaxArka Sujat ( 45.88\%) exhibited highest positive significant heterosis over better parents and the cross combination Kokan Harita×Arka Sujat 
(52.36\%) recorded significantly highest positive heterosis over standard check for length of fruit. The cross combination Pusa nasdar×Swati Green (9.17\%) exhibited highest significant heterosis over better parents and the cross combination Arka sujat×Pusa Nasdar (24.76\%) recorded significantly highest positive heterosis over standard check for diameter of fruit. The cross combination Arka Sujat×Krishni-51 (47.43\%) exhibited highest significant heterosis over better parents and the cross combination Arka Sujat×Krishna-51 (53.82\%) recorded significantly highest positive heterosis over standard check for number of fruits vine ${ }^{-1}$. The cross combination Krishni-51×Jaipuri (17.87\%) exhibited highest significant heterosis over better parents and the cross combination Kokan HaritaxArka Sujat (33.06\%) recorded significantly highest positive heterosis over standard check for weight of fruit. The cross combination Ktrishna-51×Kranti- 30 (22.08\%) exhibited highest significant heterosis over better parents and the cross combination Kokan HaritaxArka Sujat (64.96\%) recorded significantly highest positive heterosis over standard check for weight of fruit vine ${ }^{-1}$. Similarly result recorded by Mole et al. (2001), Choudhary et al (2011); Reddy et al. (2013).

The cross combination Arka SujatxKrishna-51 (38.58\%) exhibited highest significant heterosis over better parents and the cross combination Arka Sujat×Krishna-51 (84.42\%) recorded significantly highest positive heterosis over standard check for weight of fruit yield ha-1. similarly, result recorded by Naliyadhara et al. (2007), Karmakar (2011), Kumar et al (2011), Lodam et al. (2014)

\section{Conclusion}

Cross combination Arka SujatxKrishna-51, Krishna51×Kranti-30 and Krishna-51×Swati Green has shown best result for maximum fruit yield $\mathrm{ha}^{-1}$ and the cross combination Kokan HaritaxJaipuri, Kokan HaritaxKranti-30, Arka Sujat×Swati Green has shown good result for yield contributing characters.

\section{Referances}

Choudhary, B.R., Pandy, S., Singh, P.K., Singh, R., 2011. Genetic divergencein hermaphrodite ridge gourd ( $L$ uffa acutungula L ). Vegetable Science 38(1), 68-72.

Hedau, N.K., Sirohi, P.S., 2004. Combining ability for yield and its contributing characters ridge gourd Prog. Horticulture 36 (I), 91-93.
Kannan, D., Aravindan, S., Mishra, V., Kumar, P., Khan, A.H., 2015. Heterosis Studies in Ridge Gourd (Luffa acutangula L. Roxb.) in $F_{1}$ Hybrid Involving Male Sterile Indian Horticulture Journal 5(1/2), 32-35.

Karmakar, P., 2011. Studies on heterosis and inheritance of hermaphorditism in ridge gourd ( Luffa acutangula Roxb). Phd. Thesis. IARI, New Delhi

Kumar, R., Munshi, A.D., Behera, T.K., Kumar, R., Sureja, A.K., 2011. Estimates of genetic components of variation in sponge gourd. Journal of Vegetation Science 39(2), 214-217.

Lodam, V.A., Patil, P.P., Desai, D.T., 2014. Exploitation of hybrid vigour in ridge gourd (Luffa acutangula L.) Electronic Journal of Plant Breeding 5(4), 792-796.

Mole, T. J., Devi, S.N., Rajan, S., Sadhankumar, P.G., 2001. Heterosis and combining ability in ridge gourd (Luffa acutangula Roxb.). Journal of Vegetation Science 28(2), 165-167.

Naliyadhara, M.V., Dhaduk, L.K., Barad, A.V., Purohit and Vachhani, J.H., 2007. Heterosis for fruit yield and its components in sponge gourd (Luffa cylindrical L. ). National Journal of Plant Improvement 9(2), 132-135.

Narasannavar, A.R., Gasti, V.D., Shantappa, T., Mulge, R., Allolli, T.B., Thammaiahn, 2014. Heterosis studies in ridge gourd ( Luffa acutangula (L.) Roxb.). Karnataka Journal of Agricultural Sciences 27 (1), 47-51.

Panse, V.G., Sukhatme, P.V., 1985. Statistical Methods for Agricultural Workers, $4^{\text {th }}$ Edn.ICAR, New Delhi.

Radha Rani, K., 2014. Dillel analysis for different horiclture traits in bitter gourd. An International Journal by Society for Tropical Plant Research 1(2), 60-64.

Reddy, K.S., Rao, M.R., 1984. Studis in heritability, genetic advance and characyer association in ridge gourd. South Indian horticulture 32 (2), 97-100.

Shaha, S.R., Kale, P.N. 2003. Diallel analysis for combining ability in ridge gourd. Journal Of Maharashtra Agricultural Universities 28(3), 252-254.

Singh, H., Swarup, V., 1971. Exploitation of hybrid vigour in vegetable. I.C.A.R. Technical bulletin (Agri.) 30, 40.

Turner, J. H., 1953. A study of heterosis in upland cotton-I. yield of hybrid compared with varieties. II. Combining ability and inbreeding effects. Agronomy Journal 43, 487-490. 